\title{
Muscle Strength and Muscle Mass in Older Patients during Hospitalization: The EMPOWER Study
}

\author{
Jeanine M. Van Ancum ${ }^{a}$ Kira Scheerman ${ }^{b}$ Vincent D. Pierik ${ }^{b} \quad$ Siger T. Numans ${ }^{b}$ \\ Sjors Verlaan ${ }^{b, c}$ Hanne E. Smeenk ${ }^{d}$ Monique Slee-Valentijn ${ }^{\text {e }}$ \\ Roeliene C. Kruizinga ${ }^{b}$ Carel G.M. Meskers ${ }^{f} \quad$ Andrea B. Maier $^{a} g$ \\ a Department of Human Movement Sciences, Vrije Universiteit Amsterdam, Amsterdam Movement Sciences, and \\ bSection of Gerontology and Geriatrics, Department of Internal Medicine, VU University Medical Center, \\ Amsterdam Movement Sciences, Amsterdam, ' Nutricia Research, Nutricia Advanced Medical Nutrition, Utrecht, \\ dGERION, Department of General Practice and Elderly Care Medicine, VU University Medical Center, ${ }^{e}$ Center of \\ Excellence in Geriatric Rehabilitation, Cordaan, and ${ }^{\mathrm{f}}$ Department of Rehabilitation Medicine, VU University Medical \\ Center, Amsterdam Movement Sciences, Amsterdam, The Netherlands; ${ }^{9}$ Department of Medicine and Aged Care, \\ Royal Melbourne Hospital, University of Melbourne, Melbourne, VIC, Australia
}

\section{Keywords}

Geriatric medicine $\cdot$ Muscle $\cdot$ Muscle strength $\cdot$ Age-related changes $\cdot$ Old age $\cdot$ Risk factors

\begin{abstract}
Background: Low muscle strength and muscle mass are associated with an increased length of hospital stay and higher mortality rate in inpatients. To what extent hospitalization affects muscle strength and muscle mass is unclear. Objective: We aimed to assess muscle strength and muscle mass at admission and during hospitalization in older patients and its relation with being at risk of geriatric conditions. Methods: The EMPOWER study included patients aged 70 years and older, admitted to 4 wards of the VU University Medical Center in the Netherlands between April and December 2015. At admission, patients were screened for being at risk of 4 geriatric conditions: delirium, falls, malnutrition, and functional disability. At admission and at discharge, muscle strength and muscle mass were assessed. Results: A total of 373 patients (mean age, standard deviation [SD]: 79.6, 6.38 years)
\end{abstract}

\section{KARGER}

E-Mail karger@karger.com www.karger.com/ger
(C) 2017 The Author(s)

Published by S. Karger AG, Basel

Karger

Open access

This article is licensed under the Creative Commons AttributionNonCommercial-NoDerivatives 4.0 International License (CC BYNC-ND) (http://www.karger.com/Services/OpenAccessLicense).
Usage and distribution for commercial purposes as well as any distribution of modified material requires written permission. were included at admission, and 224 patients (mean age, SD: 80.1, 6.32 years) at discharge. At admission, lower muscle strength in both female and male patients and low muscle mass in male patients were associated with being at risk of a higher cumulative number of geriatric conditions. Muscle strength increased during hospitalization, but no change in muscle mass was observed. Changes in muscle measures were not associated with being at risk of geriatric conditions. Discussion: Older patients with lower muscle strength and muscle mass at admission were at risk of a higher cumulative number of geriatric conditions. However, being at risk of geriatric conditions did not forecast further decrease in muscle strength and muscle mass during hospitalization

(C) 2017 The Author(s)

Published by S. Karger AG, Basel

\section{Introduction}

Low muscle strength and muscle mass are highly prevalent in older patients at time of admission [1,2] and are associated with dependency in activities of daily living

Andrea B. Maier

Department of Medicine, The Royal Melbourne Hospital, The University of Melbourne Clinical Sciences Building, Royal Parade

Parkville, VIC 3010 (Australia)

E-Mail andrea.maier@mh.org.au 
(ADL), a longer length of stay, and higher mortality rate after discharge $[2,3]$. Muscle strength and muscle mass are predominantly affected by physical inactivity $[4,5]$. In healthy older persons, 10 days of enforced bedrest caused a loss of $10 \%$ lean leg mass $[6,7]$.

During hospitalization, older patients spend most of their time lying in bed $[8,9]$. This may affect muscle strength and muscle mass and add to aforementioned adverse outcomes. In support of this hypothesis, a previous study showed that community-dwelling older people, who had been hospitalized during the previous year, had significant lower muscle strength and muscle mass compared to people who had not been admitted [10]. We recently performed a meta-analysis on the change of muscle strength and muscle mass in older patients during hospitalization and found evidence for a decrease in muscle strength and muscle mass in electively admitted patients, whereas no significant change was found in acutely admitted patients [11]. However, the included studies were small and did not identify individual risk factors for a decrease in muscle strength and muscle mass. Due to an impaired physiological reserve, patients at risk of geriatric conditions like delirium, falls, malnutrition, and functional disability might have an even greater risk of physical deterioration compared to patients not at risk [12-15].

This study aimed to evaluate muscle strength and muscle mass at admission and during hospitalization in an inception cohort of patients aged 70 years and older, and its association with being at risk of geriatric conditions at admission.

\section{Materials and Methods}

\section{Study Design}

The Evaluation of Muscle parameters in a Prospective cohort of Older patients at clinical Wards Exploring Relations with bed rest and malnutrition (EMPOWER) study is an observational, prospective, and longitudinal inception cohort study conducted from April until December 2015 at the VU university medical center in Amsterdam, the Netherlands. A total of 838 patients aged 70 years and older who were admitted to the internal medicine, acute admission, trauma, and orthopedic wards were considered eligible for participation in the study. All patients included in the study had to be able and willing to sign informed consent. Patients were excluded when they were nursed in air-pressure isolation rooms, suffering from terminal illness (as stated by their responsible specialist), expected to be discharged within $24 \mathrm{~h}$, or could not be assessed within $48 \mathrm{~h}$ after admission.

A total of 378 patients were included in the EMPOWER study (Fig. 1). The assessment at admission included general characteristics, geriatric risk conditions and measurement of muscle strength and muscle mass. If patients were discharged within 1 day after the first assessment, they were excluded from the inhospital follow-up assessment. In-hospital follow-up was performed 7 days after the first assessment or earlier at the day of discharge in 224 patients (59.3\%). The in-hospital follow-up assessment included re-measurement of muscle strength and muscle mass. The main reason for missing follow-up assessments were discharges within 1 day after the first assessment $(n=72)$. Seven patients died before a follow-up measurement could be performed. The study was approved by the Medical Ethics Committee of the VU University Medical Center and all included patients signed informed consent.

\section{Clinical Measures}

Admission diagnosis, medical history, and medication use were obtained from medical records. Other characteristics were gathered at bedside and included alcohol intake (current use, yes or no), smoking behavior (current use, yes or no), use of walking aid, living situation, 6-item Cognitive Impairment Test (6-CIT, score ranging from 0 to 28) [16], Numeric Rating Scale for pain (NRS; score ranging from 0 to 10), and Functional Ambulatory Categories (FAC; score ranging from 0 to 5) [17]. Height in $\mathrm{cm}$ was estimated using knee height in $\mathrm{cm}$, as proposed by the Longitudinal Aging Study Amsterdam (LASA) formula (male $=74.48+$ $[2.03 \times$ knee height $]-[0.15 \times$ age $]$, female $=68.74+[2.07 \times$ knee height $]-[0.16 \times$ age $])$. Weight in $\mathrm{kg}$ was measured using a weighting chair. If patients were bedbound, an estimate was obtained from the patient or relative. Body mass index was calculated as weight/height in meters ${ }^{2}\left(\mathrm{~kg} / \mathrm{m}^{2}\right)$.

\section{Risk of Geriatric Conditions}

All patients were screened for risk of 4 geriatric conditions. Risk of delirium was identified by asking for self-perceived memory problems, the need of care in the $24 \mathrm{~h}$ prior to admission and delirium during previous hospitalization [18] (score ranging from 0 to 3 , at risk for delirium when score is $>0$ ). Risk of falls was identified by questioning the patient on fall incidence in previous 6 months [18] (at risk if answered positive). Risk of malnutrition was measured using the Short Nutritional Assessment Questionnaire (SNAQ) [19] (score ranging from 0 to 7, at risk when score is $>1$ ). Risk of functional disability was measured using Katz-ADL [20] (score ranging from 0 to 6 , at risk when score is $>1$ ). Each risk of a geriatric condition was summed resulting in being at risk of a cumulative number of geriatric conditions (score ranging from 0 to 4$)$.

\section{Muscle Measures}

\section{Muscle Strength}

Hand grip strength (HGS), shown to be a good representative of overall muscle strength in community-dwelling older people [21], was measured twice for each hand, using a Jamar Hydraulic Handheld Dynamometer (Sammons Preston Inc., Bolingbrook, IL, USA) and expressed in $\mathrm{kg}$. Patients were asked to squeeze maximally, encouraged by the assessor. The maximum score of either the left or right side was used for analyses. Patients were in upright position with the elbow flexed $90^{\circ}$ and the elbows unsupported. If patients were unable to get out of bed, HGS was measured in semisupine position in an angle of approximately $30^{\circ}$ with the elbows unsupported. During the follow-up assessment, patients were positioned in the same way as during the first assessment.
508

Gerontology 2017;63:507-514

DOI: $10.1159 / 000478777$
Van Ancum et al. 


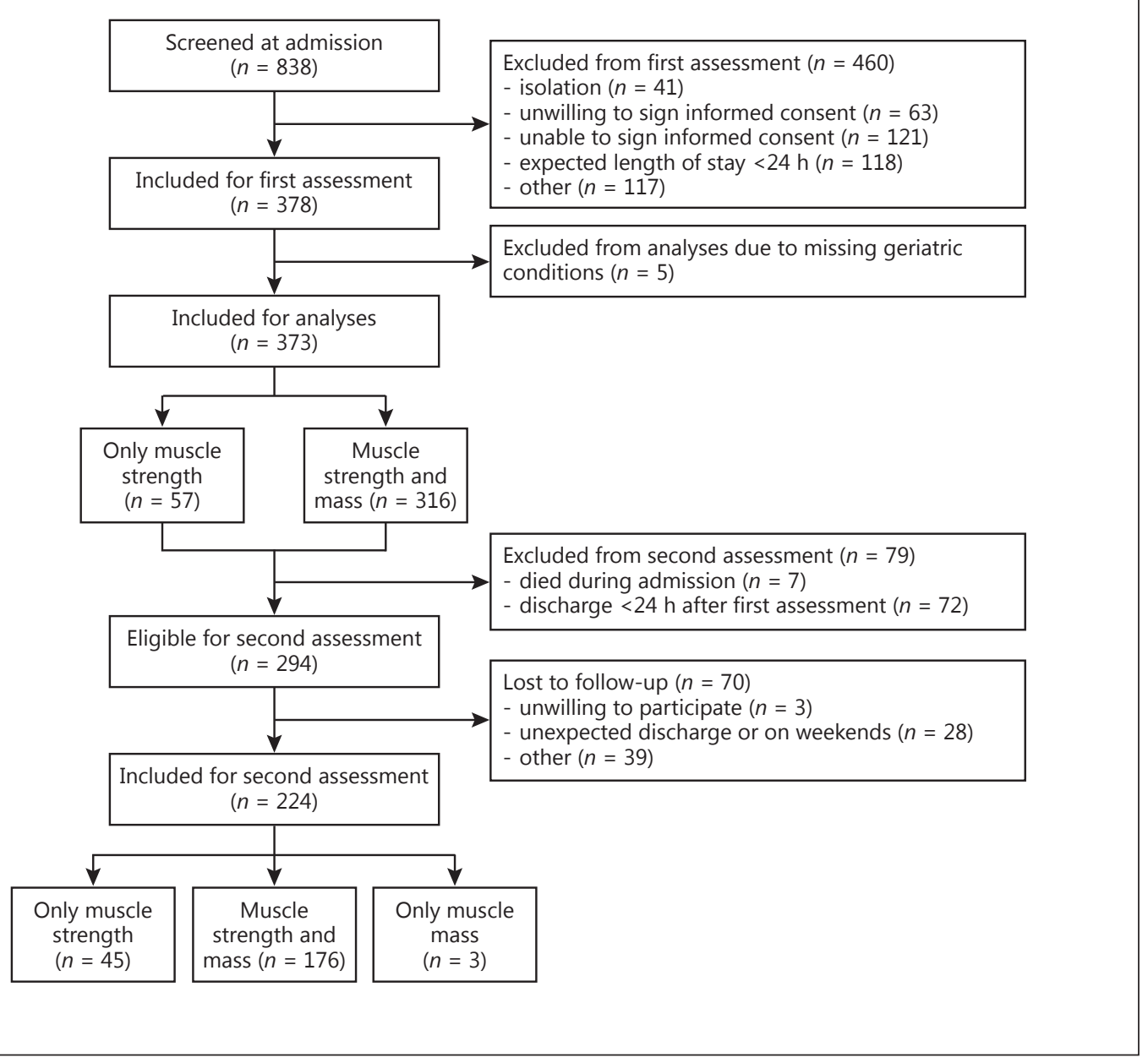

Fig. 1. Flowchart, number of patients included for each assessment.

Muscle Mass

Muscle mass was measured using direct segmental multifrequency bioelectrical impedance analysis (DSM-BIA; In-Body S10; Biospace Co., Ltd, Seoul, Korea) [22] and expressed as: (1) skeletal muscle mass (SMM) in $\mathrm{kg}$, (2) appendicular lean mass (ALM) in $\mathrm{kg}$, (3) SMM index (SMI: SMM/height ${ }^{2}$ ) in $\mathrm{kg} / \mathrm{m}^{2}$, (4) relative SMM $(\mathrm{SMM} /$ weight in $\mathrm{kg} \times 100)$ in percentage $(\%)$ and $(5)$ relative $\operatorname{ALM}(\mathrm{ALM} /$ weight in $\mathrm{kg} \times 100)$ in $\%$. At the time of measurement, patients were in a supine position, with extremities straightened and not touching their core. Patients were asked not to move during the measurement. Exclusion criteria for DSM-BIA measurement were a pacemaker or an implantable cardioverter-defibrillator, plasters or bandages that could not be removed from the positioning place of the electrodes, or amputated arm and/or leg. In 57 patients, DSM-BIA was omitted due to presence of contraindications (Fig. 1).

Muscle Strength and Muscle Mass in Older Patients

\section{Statistical Analysis}

Descriptive statistics for continuous variables with a normal distribution were presented as mean with standard deviation (SD). Variables with a skewed distribution (non-Gaussian) were presented as median with interquartile range. Categorical variables were presented as numbers with percentage, $n(\%)$. Five patients of whom data on one or more of the risk of geriatric conditions were missing, were excluded from analyses. Characteristics of patients included and excluded from or lost to follow-up were compared using independent-samples $t$ test (normal distribution), Mann-Whitney U test (skewed distribution) or $\chi^{2}$ test (categorical variables). All further analyses were stratified by sex.

Linear regression analyses were used to study the association of HGS and muscle mass with being at risk of geriatric conditions at hospital admission and presented as beta and 95\% confidence interval. Analyses were performed unadjusted (crude model) and adjusted for age and number of comorbidities, height (in model of HGS), or weight (in model of relative muscle mass). Two pa- 
Table 1. Patient characteristics

\begin{tabular}{|c|c|}
\hline Characteristics & $\begin{array}{l}\text { Total } \\
(n=373)\end{array}$ \\
\hline Age, years & $79.6 \pm 6.38$ \\
\hline Sex, female & $182(48.8)$ \\
\hline Use of walking aid & $198(53.4)$ \\
\hline Living independently & $336(91.1)$ \\
\hline Weight, kg & $73.3 \pm 17.1$ \\
\hline Height, $\mathrm{cm}$ & $169 \pm 9.45$ \\
\hline $\mathrm{BMI}$ & $25.8 \pm 5.78$ \\
\hline Current smoking & $39(10.7)$ \\
\hline Alcohol use & $146(40.2)$ \\
\hline Elective admission & $58(15.5)$ \\
\hline \multicolumn{2}{|l|}{ Reason for admission } \\
\hline Gastrointestinal & $49(13.1)$ \\
\hline Infectious & $111(29.8)$ \\
\hline Surgical & $47(12.6)$ \\
\hline Trauma & $58(15.5)$ \\
\hline Other & $108(29.0)$ \\
\hline Polypharmacy ${ }^{\mathrm{a}}$ & $322(86.6)$ \\
\hline Comorbidities $^{\mathrm{b}}$ & $328(88.4)$ \\
\hline At risk of delirium & $222(59.5)$ \\
\hline Falls in previous 6 months & $169(45.3)$ \\
\hline SNAQ score $>1$ & $129(34.6)$ \\
\hline ADL score $>1$ & $153(41.0)$ \\
\hline 6-item CIT & $4(0-8)$ \\
\hline NRS score on pain & $2(0-5)$ \\
\hline FAC score & $2(0-5)$ \\
\hline Length of stay, days & $5.01(2.91-7.78)$ \\
\hline Time between measurements, days & $4.99(3.00-6.95)$ \\
\hline
\end{tabular}

All variables are presented as $n(\%)$, mean \pm standard deviation, or median (interquartile range). BMI, body mass index; SNAQ, short nutritional assessment questionnaire; ADL, KATZ activities of daily living; CIT, cognitive impairment test; NRS, numerical rating scale; FAC, functional ambulation categories. ${ }^{\text {a }}$ Number of medications $>4 .{ }^{\mathrm{b}}$ Number of comorbidities $>1$.

tients were found to deviate more than 3 SD from the mean muscle mass of the cohort and were excluded from the analyses on muscle mass.

Changes in HGS and muscle mass during hospitalization were analyzed using paired-samples $t$ tests. Linear regression analyses were used to study the change in HGS and muscle mass associated with being at risk of geriatric conditions. The same adjustments were used as for the cross-sectional analyses, with additional adjustments for HGS and muscle mass at admission and time between measurements (adjusted model 1). All analyses were performed using the Statistical Package for the Social Sciences (IBM SPSS Statistics for Windows, version 22.0; Armonk, NY, IBM Corp). Results at admission were visualized as bar charts with unstandardized predicted means using GraphPad Prism for Windows (version 7.0. GraphPad Software Inc.). $p$ values below 0.05 were considered statistically significant.

\section{Results}

Table 1 shows the characteristics of the patients included in the study. The mean age (SD) of the patients was 79.6 years (6.38). Sixty percent of the patients were at risk of delirium, $45 \%$ were at risk of falls, $35 \%$ were at risk of malnutrition, and $41 \%$ were at risk of functional disability. Fourteen percent of the patients were not at risk of any geriatric condition, 29 were at risk of one, 24 of two, 25 of three, and $6 \%$ were at risk of 4 geriatric conditions.

Table 2 shows the association of HGS and muscle mass at admission with being at risk of geriatric conditions. Lower HGS was associated with being at risk of a higher cumulative number of geriatric conditions in both female and male patients. In male patients, lower absolute SMM, absolute ALM, and SMI were associated with being at risk of a higher cumulative number of geriatric conditions. After adjustment for confounders, these results remained statistically significant and an additional association was found in male patients for lower relative SMM. No association was found for relative ALM. The adjusted results are visualized in Figure 2.

Table 3 shows the values for HGS and muscle mass at admission and the change during hospitalization. HGS, absolute ALM, and relative ALM increased significantly during hospitalization in both female and male patients. No statistically significant changes in absolute SMM, SMI, and relative SMM were found. The association of changes in HGS and muscle mass during hospitalization with being at risk of geriatric conditions is shown in Table 4. A higher increase in relative SMM was associated with being at risk of a higher cumulative number of geriatric conditions in male patients, but statistical significance was lost after adjustment for possible confounders. No other associations of change in HGS and muscle mass with being at risk of geriatric conditions were found.

\section{Discussion}

In this inception cohort of hospitalized patients aged 70 years and older, a lower HGS in female and male patients and a lower absolute and relative muscle mass in male patients were associated with being at risk of a higher cumulative number of geriatric conditions at admission. HGS, ALM, and relative ALM increased during hospitalization, but these changes were not associated with the number of geriatric conditions.

Associations of separate geriatric conditions, i.e. delirium, falls, malnutrition, and functional disability, with 
Table 2. Hand grip strength and muscle mass of patients at admission associated with being at risk of geriatric conditions, stratified by sex

\begin{tabular}{|c|c|c|c|c|c|c|}
\hline Risk of geriatric conditions $\mathrm{s}^{\mathrm{a}}$ & HGS, kg & SMM, kg ${ }^{\mathrm{b}}$ & $\mathrm{ALM}, \mathrm{kg}^{\mathrm{b}}$ & SMI, $\mathrm{kg} / \mathrm{m}^{2 \mathrm{~b}}$ & Relative SMM, \% ${ }^{\mathrm{b}}$ & Relative ALM, \% ${ }^{\mathrm{b}}$ \\
\hline \multicolumn{7}{|l|}{ Female $(n=182)$} \\
\hline Crude, B & $-1.25(-1.93,0.57)$ & $-0.37(-0.86,0.12)$ & $-0.23(-0.70,0.24)$ & $-0.10(-0.26,0.06)$ & $-0.53(-1.27,0.20)$ & $-0.39(-0.92,0.14)$ \\
\hline Adjusted, B & $-0.90(-1.55,0.25)$ & $-0.26(-0.77,0.25)$ & $-0.16(-0.64,0.33)$ & $-0.08(-0.24,0.09)$ & $-0.33(-0.92,0.27)$ & $-0.24(-0.73,0.26)$ \\
\hline \multicolumn{7}{|l|}{ Male $(n=191)$} \\
\hline Crude, $\mathrm{B}$ & $-3.61(-4.72,2.49)$ & $-2.10(-2.79,1.41)$ & $-1.44(-2.03,0.85)$ & $-0.59(-0.77,0.41)$ & $-0.52(-1.17,0.13)$ & $-0.17(-0.69,0.36)$ \\
\hline Adjusted, B & $-2.26(-3.31,1.22)$ & $-1.71(-2.42,0.99)$ & $-1.18(-1.79,0.56)$ & $-0.55(-0.74,0.36)$ & $-0.92(-1.57,0.27)$ & $-0.39(-0.95,0.17)$ \\
\hline
\end{tabular}

Figures in parentheses indicate 95\% CI. Statistically significant results are presented in bold. HGS, hand grip strength; SMM, skeletal muscle mass; ALM, appendicular lean mass;

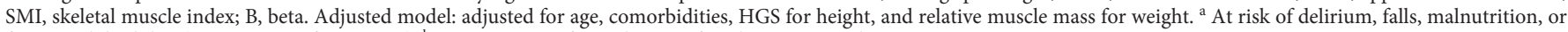
functional disability (score ranging from 0 to 4 ). ${ }^{\mathrm{b}}$ For measures of muscle mass: female $n=158$, male $n=156$.

Fig. 2. Hand grip strength and muscle mass of patients at admission associated with being at risk of geriatric conditions, stratified by sex. Bars indicate predicted means, adjusted for age, comorbidities, HGS for height, and relative muscle mass for weight. Error bars indicate 1 standard error. ${ }^{*} p \leq$ $0.05, * * p<0.01, * * * p<0.001$. HGS, hand grip strength; SMM, skeletal muscle mass; ALM, appendicular lean mass; SMI, SMM index. Risk of geriatric conditions: at risk of delirium, falls, malnutrition, or functional disability (score ranging from 0 to 4 ).

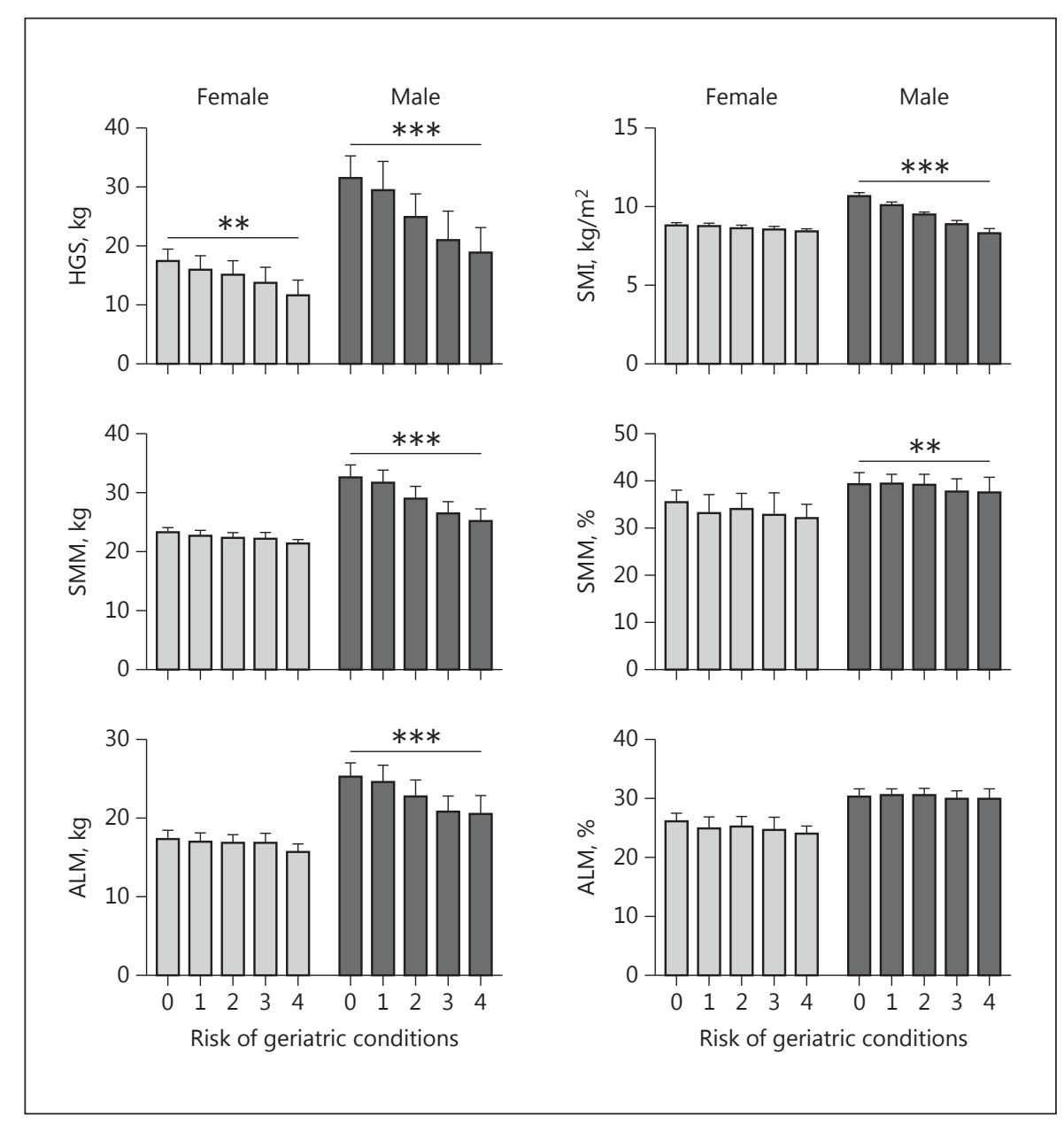

muscle measures were previously studied in specific populations. Low muscle mass was associated with increased prevalence of delirium in older participants living in nursing homes [12]. Low muscle mass and muscle strength were associated with increased prevalence of falls in the past year in community-dwelling older adults
[13] and in older outpatients [23], and with dependence in ADL and malnourishment in older hospitalized patients [14]. A recent systematic review summarized the negative consequences of sarcopenia, including falls, functional limitations, functional decline, and mortality in various older populations [24]. We recently showed 
Table 3. Handgrip strength and muscle mass of patients at admission and in-hospital change, stratified by sex

\begin{tabular}{|c|c|c|c|c|c|c|}
\hline & \multicolumn{3}{|l|}{ Female } & \multicolumn{3}{|l|}{ Male } \\
\hline & $\begin{array}{l}\text { admission } \\
(n=120)\end{array}$ & $\begin{array}{l}\text { discharge } \\
(n=120)\end{array}$ & $p$ value & $\begin{array}{l}\text { admission } \\
(n=101)\end{array}$ & $\begin{array}{l}\text { discharge } \\
(n=101)\end{array}$ & $p$ value \\
\hline HGS, kg & $14.6(5.32)$ & $15.5(5.20)$ & 0.017 & $25.6(9.56)$ & $26.5(9.35)$ & 0.033 \\
\hline SMM, $\mathrm{kg}^{\mathrm{a}}$ & $22.4(3.75)$ & $22.5(3.91)$ & 0.958 & $29.2(5.08)$ & $29.1(5.00)$ & 0.811 \\
\hline $\mathrm{ALM}, \mathrm{kg}^{\mathrm{a}}$ & $16.7(3.49)$ & $17.2(3.68)$ & 0.011 & $22.8(4.30)$ & $23.2(4.42)$ & 0.033 \\
\hline SMI, $\mathrm{kg} / \mathrm{m}^{2 \mathrm{a}}$ & $8.61(1.20)$ & $8.61(1.26)$ & 0.985 & $9.61(1.42)$ & $9.60(1.41)$ & 0.814 \\
\hline Relative SMM, \% ${ }^{\mathrm{a}}$ & $34.5(5.84)$ & $34.6(6.13)$ & 0.930 & $39.3(4.90)$ & $39.3(5.08)$ & 0.905 \\
\hline Relative ALM, $\%^{\mathrm{a}}$ & $25.5(4.34)$ & $26.5(5.51)$ & 0.005 & $30.6(3.53)$ & $31.2(4.14)$ & 0.022 \\
\hline
\end{tabular}

All variables are presented as mean (standard deviation). Statistically significant results are presented in bold. HGS, hand grip

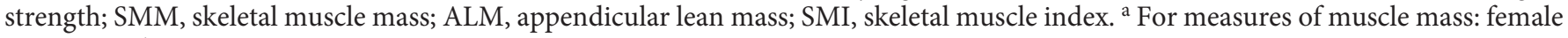
$n=101$, male $n=78$.

Table 4. Change in handgrip strength and muscle mass of patients associated with being at risk of geriatric conditions, stratified by sex

\begin{tabular}{|c|c|c|c|c|c|c|}
\hline Risk of geriatric conditions $\mathrm{s}^{\mathrm{a}}$ & HGS, kg & $\mathrm{SMM}, \mathrm{kg}^{\mathrm{b}}$ & $\mathrm{ALM}, \mathrm{kg}^{\mathrm{b}}$ & $\mathrm{SMI}, \mathrm{kg} / \mathrm{m}^{2 \mathrm{~b}}$ & Relative SMM, \% ${ }^{\mathrm{b}}$ & Relative ALM, $\%^{\mathrm{b}}$ \\
\hline \multicolumn{7}{|l|}{ Female $(n=120)$} \\
\hline Crude, $\mathrm{B}$ & $0.45(-0.15,1.05)$ & $0.21(-0.14,0.55)$ & $0.14(-0.19,0.48)$ & $0.08(-0.06,0.22)$ & $0.35(-0.21,0.90)$ & $0.30(-0.23,0.82)$ \\
\hline Adjusted, B & $0.23(-0.34,0.80)$ & $0.08(-0.29,0.45)$ & $0.01(-0.33,0.36)$ & $0.02(-0.12,0.16)$ & $0.09(-0.48,0.67)$ & $0.13(-0.42,0.67)$ \\
\hline \multicolumn{7}{|l|}{ Male $(n=101)$} \\
\hline Crude, $\mathrm{B}$ & $-0.02(-0.73,0.70)$ & $0.30(-0.02,0.61)$ & $0.03(-0.29,0.36)$ & $0.10(-0.01,0.20)$ & $0.43(0.01,0.85)$ & $0.07(-0.37,0.51)$ \\
\hline Adjusted, B & $-0.38(-1.16,0.40)$ & $0.27(-0.10,0.64)$ & $-0.05(-0.40,0.29)$ & $0.08(-0.04,0.21)$ & $0.40(-0.10,0.90)$ & $-0.08(-0.55,0.39)$ \\
\hline
\end{tabular}

Figures in parentheses indicate 95\% confidence interval. Statistically significant results are presented in bold. HGS, hand grip strength; SMM, skeletal muscle mass; ALM, appendicular lean mass; SMI, skeletal muscle index; B, beta. Adjusted model, adjusted for age, comorbidities, HGS and muscle mass at admission, time between measurements, HGS for height at admission, and relative muscle mass for weight at admission. a At risk of delirium, falls, malnutrition, or functional disability (score ranging from 0 to 4 ). b For measures of muscle mass: female $n=101$, male $n=78$.

that lower muscle strength was associated with institutionalization, and lower muscle mass was associated with a lower likelihood of survival after hospitalization in older patients [15]. In the present study, we considered the risk of a cumulative number of geriatric conditions as a proxy of vulnerability and showed its association with lower HGS in both female and male patients, and absolute and relative muscle mass at admission in male patients. A recent study in older inpatients showed that low muscle strength and muscle mass at admission leads to an increased risk of incomplete functional recovery during inhospital rehabilitation [25], likely mediated by the increased vulnerability of these patients. Lower relative SMM at admission was only associated with being at risk of a higher cumulative number of geriatric conditions after adjusting for weight. This illustrates that weight should be taken into account, which is in line with previous research $[26,27]$. Relative ALM did not reach significance in our cohort. These findings highlight the importance of measuring muscle strength and muscle mass in clinical practice, because it provides valuable insight into the patients' risk profile.

We hypothesized that hospitalization would influence muscle strength and muscle mass negatively in older patients, especially in patients at risk of a higher cumulative number of geriatric risk conditions. However, no measurable negative impact of hospitalization was observed; HGS and absolute and relative ALM even increased during hospitalization in this cohort, and no association with being at risk of geriatric conditions was found. It should be considered that hospitalization was relatively short, and patients were mainly acutely admitted. Moreover, patients who were lost to follow-up, had on average higher HGS, SMM, and ALM. The increase in HGS during hospitalization might be due to an influence of pain, low energy levels, and fatigue at admission $[28,29]$ and the recovery from it during hospital stay. An increase in absolute and relative ALM was not ex- 
pected to occur within the timeframe of this study [30] and may be due to change of hydration status (dehydration at admission and fluid resuscitation during hospitalization), which might have been unintentionally measured by the BIA. The aforementioned issue may mask a decrease in muscle mass during hospitalization. These findings are partly in line with our recent systematic review and meta-analysis, showing a trend towards increased HGS and no change in muscle mass in acutely admitted patients during hospitalization [11]. An important next step in future research is to include followup measurements of muscle strength and muscle mass to evaluate the total impact of hospitalization on muscle health.

\section{Sex Differences}

Muscle mass at admission was lower in female compared to male patients and was not associated with being at risk of geriatric conditions in females. Opposite to our findings, studies on single geriatric conditions showed an association in both females and males between malnutrition [14], delirium [12], and low absolute muscle mass. These studies used sex specific cut-off points for sarcopenia, defined as a combination of low absolute muscle mass and low muscle performance, instead of a continuous measure of muscle mass.

\section{Strengths and Limitations}

This is the first study reporting repeatedly measured muscle strength and muscle mass in a large inception cohort of older patients, without major exclusion criteria. Being at risk of geriatric conditions was assessed using tools that were previously validated in older patients [18-20]. Use of BIA measurements may be hampered by hydration state and change in hydration state over time. There was loss to follow-up during hospitalization, mostly due to frequent short hospital stay or sudden discharge. This may have introduced selection bias in the results; however, we believe that the present inception cohort is typically reflecting the group of older patients at risk for decline in muscle health during hospital stay.

\section{Conclusion}

In this large inception cohort of hospitalized patients aged 70 years and older, lower muscle strength at admission was associated with being at risk of a higher cumulative number of geriatric conditions in female and male patients. Lower muscle mass at admission was associated with being at risk of a higher cumulative number of geriatric conditions in male patients only. Muscle strength increased during hospitalization, while no significant change in absolute and relative SMM was observed. No associations of the change in muscle measures with being at risk of geriatric risk conditions were found. This study highlights the importance of measuring muscle parameters in clinical practice. During short-term hospitalization, no effect on muscle was found, but future studies with long-term follow-up are needed to be able to draw conclusions.

\section{Acknowledgements}

The authors would like to thank A. Verburg and H.M.D. Nagtzaam for their assistance in including patients.

\section{Disclosure Statement}

The authors declare that they have no conflicts of interest.

\section{Funding Sources}

This work was supported by the seventh framework program MYOAGE (HEALTH-2007-2.4.5-10) and European Union's Horizon 2020 research and innovation programme $(689238,675003)$, and Nutricia Research, Nutricia Advanced Medical Nutrition, The Netherlands.

References

1 Cerri AP, Bellelli G, Mazzone A, Pittella F, Landi F, Zambon A, Annoni G: Sarcopenia and malnutrition in acutely ill hospitalized elderly: prevalence and outcomes. Clin Nutr 2015;34:745-751.

2 Gariballa S, Alessa A: Sarcopenia: prevalence and prognostic significance in hospitalized patients. Clin Nutr 2013;32:772-776.

3 Peterson SJ, Braunschweig CA: Prevalence of sarcopenia and associated outcomes in the clinical setting. Nutr Clin Pract 2015;31:4048.

4 Bautmans I, Van Puyvelde K, Mets T: Sarcopenia and functional decline: pathophysiology, prevention and therapy. Acta Clin Belg 2009;64:303-316.

5 Bodine SC: Disuse-induced muscle wasting. Int J Biochem Cell Biol 2013;45:2200-2208.

6 Kortebein P, Ferrando A, Lombeida J, Wolfe R, Evans WJ: Effect of 10 days of bed rest on skeletal muscle in healthy older adults. JAMA 2007;297:1772-1774. 
7 Drummond MJ, Dickinson JM, Fry CS, Walker DK, Gundermann DM, Reidy PT, Timmerman KL, Markofski MM, PaddonJones D, Rasmussen BB, Volpi E: Bed rest impairs skeletal muscle amino acid transporter expression, mTORC1 signaling, and protein synthesis in response to essential amino acids in older adults. Am J Physiol Endocrinol Metab 2012;302:E1113-E1122.

8 Bodilsen AC, Pedersen MM, Petersen J, Beyer N, Andersen O, Smith LL, Kehlet H, Bandholm T: Acute hospitalization of the older patient: changes in muscle strength and functional performance during hospitalization and 30 days after discharge. Am J Phys Med Rehabil 2013;92:789-796.

9 Brown CJ, Redden DT, Flood KL, Allman RM: The underrecognized epidemic of low mobility during hospitalization of older adults. J Am Geriatr Soc 2009;57:1660-1665.

10 Alley DE, Koster A, Mackey D, Cawthon P, Ferrucci L, Simonsick EM, Yu B, Hardy S, Goodpaster B, Sarkisian C, Houston DK, Kritchevsky SB, Cummings S, Lee JS, Tylavsky FA, Newman A, Harris T: Hospitalization and change in body composition and strength in a population-based cohort of older persons. J Am Geriatr Soc 2010;58:20852091.

11 Van Ancum JM, Scheerman K, Jonkman NH, Smeenk HE, Kruizinga RC, Meskers CG, Maier $\mathrm{AB}$ : Change in muscle strength and muscle mass in older hospitalized patients: a systematic review and meta-analysis. Exp Gerontol 2017;92:34-41.

12 Culp KR, Cacchione PZ: Nutritional status and delirium in long-term care elderly individuals. Appl Nurs Res 2008;21:66-74.

13 Clynes MA, Edwards MH, Buehring B, Dennison EM, Binkley N, Cooper C: Definitions of sarcopenia: associations with previous falls and fracture in a population sample. Calcif Tissue Int 2015;97:445-452.
14 Sousa AS, Guerra RS, Fonseca I, Pichel F, Amaral TF: Sarcopenia among hospitalized patients - a cross-sectional study. Clin Nutr 2015;34:1239-1244.

15 Verlaan S, Van Ancum JM, Pierik VD, van Wijngaarden J, Scheerman K, Meskers CG, Maier AB: Muscle measures and nutritional status at hospital admission predict survival and independent living of older patients - the EMPOWER study. J Frailty Aging 2017;6: 161-166.

16 Abdel-Aziz K, Larner AJ: Six-item cognitive impairment test (6CIT): Pragmatic diagnostic accuracy study for dementia and MCI. Int Psychogeriatr 2015;27:991-997.

17 Holden MK, Gill KM, Magliozzi MR, Nathan J, Piehl-Baker L: Clinical gait assessment in the neurologically impaired. Reliability and meaningfulness. Phys Ther 1984;64:35-40.

18 Oud FM, de Rooij SE, Schuurman T, Duijvelaar KM, van Munster BC: Predictive value of the VMS theme "frail elderly": delirium, falling and mortality in elderly hospital patients (in Dutch). Ned Tijdschr Geneeskd 2015; 159:A8491.

19 Kruizenga HM, Seidell JC, de Vet HC, Wierdsma NJ, van Bokhorst-de van der Schueren MA: Development and validation of a hospital screening tool for malnutrition: the short nutritional assessment questionnaire (SNAQ). Clin Nutr 2005;24:75-82.

20 Katz S, Ford AB, Moskowitz RW, Jackson BA, Jaffe MW: Studies of illness in the aged. The index of ADL: a standardized measure of biological and psychosocial function. JAMA 1963;185:914-919.

21 Rantanen T, Era P, Heikkinen E: Maximal isometric strength and mobility among 75-yearold men and women. Age Ageing 1994;23: 132-137.

22 Ling $\mathrm{CH}$, de Craen AJ, Slagboom PE, Gunn DA, Stokkel MP, Westendorp RG, Maier AB: Accuracy of direct segmental multi-frequency bioimpedance analysis in the assessment of total body and segmental body composition in middle-aged adult population. Clin Nutr 2011;30:610-615.
23 Tan LF, Lim ZY, Choe R, Seetharaman S, Merchant R: Screening for frailty and sarcopenia among older persons in medical outpatient clinics and its associations with healthcare burden. J Am Med Dir Assoc 2017;18: 583-587.

24 Beaudart C, Zaaria M, Pasleau F, Reginster JY, Bruyere O: Health outcomes of sarcopenia: a systematic review and meta-analysis. PLoS One 2017;12:e169548.

25 Landi F, Calvani R, Ortolani E, Salini S, Martone AM, Santoro L, Santoliquido A, Sisto A, Picca A, Marzetti E: The association between sarcopenia and functional outcomes among older patients with hip fracture undergoing in-hospital rehabilitation. Osteoporos Int 2017;28:1569-1576.

26 Bijlsma AY, Meskers MC, Molendijk M, Westendorp RG, Sipila S, Stenroth L, Sillanpaa E, McPhee JS, Jones DA, Narici M, Gapeyeva H, Paasuke M, Seppet E, Voit T, Barnouin Y, Hogrel JY, Butler-Browne G, Maier AB: Diagnostic measures for sarcopenia and bone mineral density. Osteoporos Int 2013;24:26812691.

27 Szulc P, Beck TJ, Marchand F, Delmas PD: Low skeletal muscle mass is associated with poor structural parameters of bone and impaired balance in elderly men - the MINOS study. J Bone Miner Res 2005;20:721-729.

28 Boter H, Manty M, Hansen AM, Hortobagyi $\mathrm{T}$, Avlund K: Self-reported fatigue and physical function in late mid-life. J Rehabil Med 2014;46:684-690.

29 Rudy TE, Lieber SJ, Boston JR: Functional capacity assessment: influence of behavioral and environmental factors. J Back Musculoskelet Rehabil 1996;6:277-288.

30 Shavlakadze T, McGeachie J, Grounds MD: Delayed but excellent myogenic stem cell response of regenerating geriatric skeletal muscles in mice. Biogerontology 2010;11:363-376. 